\title{
Predictive Risk Factors for Fear of Hypoglycemia and Anxiety-Related Emotional Disorders among Adolescents with Type 1 Diabetes
}

\author{
Ayman A. Al Hayek Asirvatham A. Robert Rim B. Braham Besher A. Issa \\ Fahad S. Al Sabaan \\ Department of Endocrinology and Diabetes, Diabetes Treatment Center, Prince Sultan Military Medical City, \\ Riyadh, Saudi Arabia
}

\section{Key Words}

Type 1 diabetes mellitus $\cdot$ Fear of hypoglycemia $\cdot$ Anxiety

\begin{abstract}
Objective: To explore the fear of hypoglycemia $(\mathrm{FOH})$ and anxiety-related emotional disorders and their risk factors among adolescents with type 1 diabetes mellitus (T1DM). Subjects and Methods: A cross-sectional study was conducted among 187 adolescents (aged 13-18 years; 92 males, 95 females) with T1DM at the Diabetes Treatment Center, Prince Sultan Military Medical City, Riyadh, Saudi Arabia, from June 2013 to February 2014. The participants were interviewed using FOH and Screen for Child Anxiety-Related Disorders (SCARED) scales. Results: Females had significantly higher scores on all FOH and SCARED subscales compared to males. The mean scores for many subscales of FOH and SCARED were higher in the older age group (16-18 years), in those under multiple-dose injection (MDI) treatment (compared with the insulin pump treatment), and in those with a longer duration of T1DM. Similarly, significant differences were observed in those with high frequencies of hypoglycemia, passing out, hypoglycemia while asleep and awake, and hypoglycemia in front of friends and at school. Regression analysis revealed that higher age, female gender, MDI treatment,
\end{abstract}

longer duration of T1DM, higher frequencies of hypoglycemia, passing out, hypoglycemia while asleep and awake, and hypoglycemia in front of friends and at school were the risk factors associated with the majority of the $\mathrm{FOH}$ and SCARED subscales. The behavior of the $\mathrm{FOH}$ subscale correlated with all the subscales of SCARED except the subscale of generalized anxiety disorder. Similarly, the $\mathrm{FOH}$ subscale of worry significantly correlated with all the subscales of SCARED. Conclusion: The strongest determinants of higher risk for the majority of the FOH and SCARED subscales were higher age, female gender, MDI treatment, longer duration of T1DM, higher frequency of hypoglycemia, passing out due to hypoglycemia, hypoglycemia while asleep and awake, and hypoglycemia in front of friends and at school.

(c) 2015 S. Karger AG, Basel

\section{Introduction}

Type 1 diabetes mellitus (T1DM) is one of the most common chronic, unremitting medical disorders that develops mainly during childhood or adolescence $[1,2]$. Compared to the general population, T1DM is associated with a 2- to 4 -fold increased mortality risk and requires lifelong insulin injections as well as behavioral modifica-

\begin{tabular}{|c|c|}
\hline KARGER 125 & $\begin{array}{l}\text { (C) } 2015 \text { S. Karger AG, Basel } \\
1011-7571 / 15 / 0243-0222 \$ 39.50 / 0\end{array}$ \\
\hline $\begin{array}{l}\text { E-Mail karger@karger.con } \\
\text { www.karger.com/mpp }\end{array}$ & $\begin{array}{l}\text { This is an Open Access article licensed under the terms of the } \\
\text { Creative Commons Attribution-NonCommercial } 3.0 \text { Un- } \\
\text { ported license (CC BY-NC) (www.karger.com/OA-license), } \\
\text { applicable to the online version of the article only. Distribu- } \\
\text { tion permitted for non-commercial purposes only. }\end{array}$ \\
\hline
\end{tabular}

Ayman A. Al Hayek

Senior Diabetes Educator, Department of Endocrinology and Diabetes Diabetes Treatment Center, Diabetes Education Unit

Prince Sultan Military Medical City, PO Box 7897, Riyadh 11159 (Saudi Arabia)

E-Mail ayman.alhayek@yahoo.com 
tions in order to survive $[1,2]$. Also, many children and adolescents are incapable of coping emotionally with their condition, and the T1DM causes embarrassment and hence leads to discrimination and limits social relationships [1]. Further evidence has indicated that around $90 \%$ of all patients who receive insulin have experienced hypoglycemic episodes, which is an important factor in excess mortality in patients with diabetes [3].

Despite recent developments in therapy, diabetes-related mortality among children has not dropped [4]. Compared to adults, youths with T1DM are considered at increased risk of experiencing severe hypoglycemic episodes [5]. The incidence of hypoglycemia is reported to be between 3 and 27 episodes per 100 patient-years in children with T1DM [3]. In the Diabetes Control and Complications Trial (DCCT), adolescents had considerably more severe hypoglycemic events than adults regardless of the type of treatment (conventional or intensive), and the prevalence may be even higher in younger children [5]. At a more severe level, if not treated effectively, hypoglycemia can lead to permanent sequelae and even death [6]. Research has shown that an episode of hypoglycemia can be accompanied by unpleasant symptoms from anxiety, embarrassment, palpitations, tremor, sweating, hunger, and paresthesias to neurological impairments, including behavioral changes, cognitive dysfunction, seizures, and coma $[7,8]$. Focal neurological deficits occur occasionally, although severe prolonged hypoglycemia can cause permanent brain damage [9].

Given the aversive nature of hypoglycemic episodes and the associated risks of individuals with T1DM, subjects can develop a significant fear of hypoglycemia $(\mathrm{FOH})$ that can adversely affect their quality of life, emotional well-being, diabetes management, and glycemic control [5]. While some degree of fear is considered appropriate and adaptive given the potential danger of hypoglycemia, for some individuals it may become more dangerous and problematic. For these individuals, FOH may cause increased anxiety about diabetes management, obsessive self-monitoring, deliberately keeping blood glucose levels too high, dependence on others, feelings of guilt and frustration, a sense of loss of control, embarrassment, stress, and avoidant behavior [5].

Studies have shown that the rising incidence of T1DM over the last 30 years in Saudi Arabia and the prevalence in Saudi children and adolescents is 109.5 per 100,000, which is higher than in many developed countries [10]. However, no evidence exists on the determinants of anxiety-related emotional disorders and $\mathrm{FOH}$ and their complication among Saudi T1DM subjects. Therefore, the present study aimed to explore the predictive risk factors of FOH and anxiety-related disorders among adolescents with T1DM.

\section{Subjects and Methods}

\section{Study Design and Setting}

This was a cross-sectional study conducted among 187 (92 males, 95 females) T1DM adolescents (aged 13-18 years) who had diabetes of more than 1-year duration at the Diabetes Treatment Center, Prince Sultan Military Medical City (PSMMC), Riyadh, Saudi Arabia from June 2013 to February 2014. All adolescents had a diagnosis of type 1 diabetes according to the practice guidelines of the American Diabetes Association (ADA) [11]. The study was conducted in accordance with the Declaration of Helsinki, and the protocol of the study was approved by the Research Ethics Committee, PSMMC, Riyadh, Saudi Arabia.

\section{Criteria for Selection of Patients and Data Collection}

The participants were selected according to their availability during their routine visit to the outpatient clinics. Parents and their adolescents were informed about the purpose and methods of the research verbally and in written form. Written informed consent was obtained from the parents and adolescents before the completion of study measurement. Study participants were free to withdraw from the study at any time without giving a reason. During the routine follow-up visits, sociodemographic and clinical data were collected. Adolescents were asked to complete the Arabic-translated FOH and Screen for Child Anxiety-Related Disorders (SCARED) questionnaires independently by a self-administered data collection technique [12]. The inclusion criteria were adolescents aged 13-18 years who had been followed up for at least 12 months and who did not have any other concomitant chronic disease. Exclusion criteria were adolescents who had a history of psychopathological and medical instability or visual, hearing or cognitive impairment.

\section{Glycosylated Hemoglobin}

During the study the most recent insulin dose of the participants and glycosylated hemoglobin $\left(\mathrm{HbA}_{1 \mathrm{c}}\right)$ values for blood glucose control were taken from the medical records. The $\mathrm{HbA}_{1 \mathrm{c}}$ test was the most appropriate measure of glycemic control and diagnostic test for diabetes. $\mathrm{HbA}_{1 \mathrm{c}}<7$ was considered a good control value (ADA standards of medical care in diabetes).

\section{Survey of Hypoglycemia Fear}

Hypoglycemia is defined by the American Diabetes Association and the Endocrine Society as a blood glucose value of $\leq 70$ $\mathrm{mg} / \mathrm{dl}(\leq 3.9 \mathrm{mmol} / \mathrm{l})$. We used the Hypoglycemia Fear Survey child version (HFS-C) to measure children's worries and behaviors related to their hypoglycemia. The HFS-C is a 32 -item survey that comprises a 10 -item behavior subscale (HFS-B), a 15-item worry subscale (HFS-W) and 7 questions about hypoglycemia in special situations. The items in the worry subscale measure anxiety-provoking aspects of hypoglycemia, and those in the behavior subscale measure specific behaviors to avoid hypoglycemia. The items are rated on a 5-point Likert scale ranging from 1 (never) to 5 (always). Higher scores indicate higher FOH 
Table 1. Demographic variable-wise differences of FOH and child anxiety-related disorders

\begin{tabular}{|c|c|c|c|c|c|c|c|c|}
\hline \multirow[t]{2}{*}{ Variables } & \multirow{2}{*}{$\begin{array}{l}\text { Frequency, } \\
\mathrm{n}(\%)\end{array}$} & \multicolumn{2}{|l|}{$\mathrm{FOH}$} & \multicolumn{5}{|l|}{ SCARED } \\
\hline & & behavior & worry & $\begin{array}{l}\text { panic } \\
\text { disorder }\end{array}$ & $\begin{array}{l}\text { generalized } \\
\text { anxiety } \\
\text { disorder }\end{array}$ & $\begin{array}{l}\text { separation } \\
\text { anxiety } \\
\text { disorder }\end{array}$ & $\begin{array}{l}\text { social } \\
\text { anxiety } \\
\text { disorder }\end{array}$ & $\begin{array}{l}\text { significant } \\
\text { school } \\
\text { avoidance }\end{array}$ \\
\hline \multicolumn{9}{|l|}{ Gender } \\
\hline Male & $92(49.2)$ & $2.01 \pm 0.75$ & $1.99 \pm 1.02$ & $0.65 \pm 0.49$ & $0.62 \pm 0.35$ & $0.70 \pm 0.48$ & $0.78 \pm 0.62$ & $0.29 \pm 0.36$ \\
\hline Female & $95(50.8)$ & $2.48 \pm 0.66^{*}$ & $2.65 \pm 0.68^{*}$ & $1.09 \pm 0.34^{*}$ & $0.81 \pm 0.41^{*}$ & $1.04 \pm 0.39^{*}$ & $1.11 \pm 0.43^{*}$ & $0.95 \pm 0.38^{*}$ \\
\hline \multicolumn{9}{|l|}{ Age } \\
\hline $13-15$ years & $94(50.3)$ & $1.96 \pm 0.73$ & $2.16 \pm 1.08$ & $0.86 \pm 0.49$ & $0.75 \pm 0.38$ & $0.81 \pm 0.52$ & $0.86 \pm 0.52$ & $0.62 \pm 0.49$ \\
\hline $16-18$ years & $93(49.7)$ & $2.55 \pm 0.63^{*}$ & $2.49 \pm 0.70^{*}$ & $0.88 \pm 0.46$ & $0.68 \pm 0.41$ & $0.94 \pm 0.40$ & $1.04 \pm 0.58^{*}$ & $0.63 \pm 0.50$ \\
\hline \multicolumn{9}{|l|}{ Education } \\
\hline Intermediate & $99(52.9)$ & $2.0 \pm 0.7$ & $2.2 \pm 1.12$ & $0.76 \pm 0.52$ & $0.71 \pm 0.39$ & $0.74 \pm 0.50$ & $0.73 \pm 0.50$ & $0.62 \pm 0.51$ \\
\hline Secondary & $88(47.1)$ & $2.5 \pm 0.72 *$ & $2.37 \pm 0.64$ & $1.0 \pm 0.38^{*}$ & $0.72 \pm 0.40$ & $1.0 \pm 0.38^{*}$ & $1.2 \pm 0.51^{*}$ & $0.64 \pm 0.48$ \\
\hline \multicolumn{9}{|l|}{ Exercise } \\
\hline No & $119(63.6)$ & $2.2 \pm 0.75$ & $2.36 \pm 1.01$ & $0.84 \pm 0.50$ & $0.67 \pm 0.33$ & $0.87 \pm 0.48$ & $0.93 \pm 0.63$ & $0.61 \pm 0.53$ \\
\hline Yes & $68(36.4)$ & $2.27 \pm 0.75$ & $2.27 \pm 0.77$ & $0.93 \pm 0.43$ & $0.80 \pm 0.48^{*}$ & $0.87 \pm 0.44$ & $0.98 \pm 0.38$ & $0.65 \pm 0.43$ \\
\hline \multicolumn{9}{|l|}{ Treatment type } \\
\hline Insulin pump & $36(19.3)$ & $2.20 \pm 1.06$ & $2.11 \pm 1.10$ & $0.68 \pm 0.37$ & $0.66 \pm 0.25$ & $0.59 \pm 0.32$ & $0.92 \pm 0.64$ & $0.42 \pm 0.37$ \\
\hline MDI & $151(80.7)$ & $2.28 \pm 0.56$ & $2.42 \pm 0.83^{*}$ & $0.96 \pm 0.49^{*}$ & $0.74 \pm 0.44$ & $0.99 \pm 0.47^{*}$ & $0.966 \pm 0.52$ & $0.71 \pm 0.51^{*}$ \\
\hline \multicolumn{9}{|l|}{ Duration } \\
\hline $1-7$ years & $146(78.1)$ & $2.17 \pm 0.74$ & $2.2 \pm 1$ & $0.79 \pm 0.51$ & $0.62 \pm 0.38$ & $0.79 \pm 0.46$ & $0.86 \pm 0.57$ & $0.55 \pm 0.48$ \\
\hline$>7$ years & $41(21.9)$ & $2.5 \pm 0.7^{*}$ & $2.7 \pm 0.3^{*}$ & $1.6 \pm 0.11^{*}$ & $1.05 \pm 0.25^{*}$ & $1.6 \pm 0.39 *$ & $1.2 \pm 0.34^{*}$ & $0.89 \pm 0.44^{*}$ \\
\hline \multicolumn{9}{|l|}{$\mathrm{HbA}_{1 \mathrm{c}}$} \\
\hline$>7$ & $153(81.8)$ & $2.3 \pm 0.9$ & $2.3 \pm 1.1$ & $1 \pm 0.39$ & $0.67 \pm 0.2$ & $1 \pm 0.37$ & $1.37 \pm 0.4$ & $0.47 \pm 0.53$ \\
\hline$\leq 7$ & $34(18.2)$ & $2.2 \pm 0.7$ & $2.3 \pm 0.8$ & $0.84 \pm 0.49$ & $0.73 \pm 0.42$ & $0.84 \pm 0.48$ & $0.85 \pm 0.54^{*}$ & $0.66 \pm 0.48^{*}$ \\
\hline
\end{tabular}

Groups were compared by t test. * $\mathrm{p}<0.05$ considered significant.

[13], with high levels of internal consistency $(\alpha=0.86)$ for the overall score.

The standard procedure of translation and back translation was carried out and showed good similarities to the original English version. This was done by two Arabic-speaking diabetes educators. Forward and back translations were carried out by each author. In addition, a pilot study was carried out on 10 adolescents with the Arabic-translated questionnaire.

\section{Screen for Child Anxiety-Related Emotional Disorders}

The SCARED is a self-report questionnaire with 41 items evaluating different dimensions of anxiety disorders in children - panic disorder or significant somatic symptoms, generalized anxiety disorder, separation anxiety disorder, social anxiety disorder, and significant school avoidance. The 41-item version of the SCARED was administered among the study population. Adolescents responded to items using a 3-point Likert-type scale that described the degree to which statements were true about them - not true or hardly ever true, somewhat true or sometimes true and very true or often true. The questionnaire shows high levels of internal consistency $(\alpha=0.91)$ for the overall score $[14,15]$.

\section{Statistical Analysis}

Data analysis was carried out using Microsoft Excel 2013 (Microsoft Corporation, Seattle, Wash., USA) and the Statistical Pack- age for Social Sciences, version 16 (SPSS Inc., Chicago, Ill., USA). In addition to the descriptive analysis $t$ test, one-way analysis of variance and Tukey post hoc tests were conducted to determine differences carried out for making comparisons among the test groups. Correlation between FOH and SCARED was performed using Pearson's correlation coefficient, and multivariate linear regression analysis was done to determine the variables that are associated with the FOH and SCARED subscales. A p value of $<0.05$ was considered to be statistically significant.

\section{Results}

\section{Demographic Data, FOH and Anxiety-Related}

Disorders

Differences in demographic and clinical variables of FOH and SCARED scores are shown in table 1. The mean age was $15.27 \pm 1.61$ years. Of the 187 participants, 92 (49.2\%) were male and 95 (50.8\%) female. The mean duration of diagnosis of DM was $7.1 \pm 5.2$ years. Females had significantly higher scores on all FOH and SCARED subscales compared to males ( $\mathrm{p}<0.05)$. The older age group 
(16-18 years) showed significantly higher scores on FOH (behavior and worry) and higher scores on the SCARED subscale of social anxiety disorder $(\mathrm{p}<0.05)$ than the younger age group (13-15 years). Secondary school adolescents had significantly higher scores on the FOH subscale of behavior $(\mathrm{p}<0.05)$ and the SCARED subscales of panic disorder $(\mathrm{p}<0.05)$, separation anxiety disorder $(\mathrm{p}<$ $0.05)$ and social anxiety disorder $(\mathrm{p}<0.05)$ than adolescents at intermediate educational level. Insulin pump users had significantly lower levels of worry, panic disorder, separation anxiety disorder, and significant school avoidance $(\mathrm{p}<0.05)$ than the multiple-dose injection (MDI) group. In addition, adolescents with a longer duration of diabetes (>7 years), regardless of gender, showed significantly higher scores on all subscales of FOH and SCARED. No significant difference was found in the FOH and SCARED subscales among adolescents with $\mathrm{HbA}_{1 \mathrm{c}} \leq 7$ compared to those with $\mathrm{HbA}_{1 \mathrm{c}}>7$ except the SCARED subscales of social anxiety disorder and significant school avoidance.

\section{History of Hypoglycemia}

The history of hypoglycemia among the study population is shown in table 2 . Overall, $41 \%$ of the study population had hypoglycemia $\geq 12$ times and 118 (63.1\%) agreed that low blood sugar was the big problem. Around $84 \%$ of the study population reported that they had hypoglycemia in front of friends or strangers and $80.7 \%$ reported that they had hypoglycemia when they were at school.

Mean comparisons of $\mathrm{FOH}$, anxiety-related disorders and history of hypoglycemia are shown in table 3. Adolescents with a higher frequency of hypoglycemia showed significantly higher scores on all subscales of FOH and SCARED except separation anxiety disorder compared to those with lower frequencies $(\mathrm{p}<0.05)$. The FOH subscale of behavior $(\mathrm{p}<0.05)$ and the SCARED subscale of school avoidance showed significantly higher scores in adolescents with a history of passing out due to hypoglycemia. The FOH subscale of worry and the SCARED subscale of school avoidance showed significantly higher scores in adolescents who had a past hypoglycemic episode while asleep. All FOH and SCARED subscales showed significantly higher levels in adolescents who had a hypoglycemic episode while awake. Adolescents who had hypoglycemia in front of friends or strangers showed significantly higher levels on the FOH subscales of behavior $(\mathrm{p}<0.05)$ and worry $(\mathrm{p}<0.05)$ and the SCARED subscale of generalized anxiety disorder. The FOH subscale of behavior $(\mathrm{p}<0.05)$ and the SCARED subscales of panic disorder $(\mathrm{p}<0.05)$, social anxiety disorder $(\mathrm{p}<0.05)$ and school avoidance $(\mathrm{p}<0.05)$ showed significantly
Table 2. Hypoglycemia history among the study population

\begin{tabular}{lll}
\hline $\mathrm{FOH}$ & Frequency & Percentage \\
\hline
\end{tabular}

How often in the last 12 months have you had trouble with hypoglycemic (low blood sugar) episodes?

$\begin{array}{llr}1-2 \text { times } & 14 & 7.5 \\ 3-6 & 65 & 34.9 \\ 7-11 & 31 & 16.6 \\ \geq 12 & 76 & 41.8\end{array}$

Is low blood sugar a big problem for you?

$\begin{array}{lcc}\text { Yes } & 118 & 63.1\end{array}$

$69 \quad 36.9$

Have you ever passed out due to hypoglycemia?

$\begin{array}{lll}\text { Yes } & 62 & 33.2\end{array}$

No $\quad 125 \quad 66.8$

Have you ever had a hypoglycemic episode while asleep?

$\begin{array}{lrr}\text { Yes } & 155 & 82.9\end{array}$

No $\quad 32 \quad 17.1$

Have you ever had a hypoglycemic episode while you were awake but by yourself?

$\begin{array}{lcc}\text { Yes } & 127 & 67.9 \\ \text { No } & 60 & 32.1 \\ \text { ave you ever had hypoglycemia } & \text { in } & \text { front } \\ \text { Yes } & 157 & 84 \\ \text { No } & 30 & 16 \\ \text { ave you ever had hypoglycemia } & \text { when you were at school? } \\ \text { Yes } & 151 & 80.7 \\ \text { No } & 36 & 19.3\end{array}$

higher levels in adolescents who had hypoglycemia at school.

Pearson's correlation analysis showed that the FOH subscale of behavior correlated with all the subscales of SCARED except that of generalized anxiety disorder. Similarly, the FOH subscale of worry significantly correlated with all the subscales of SCARED (table 4). Importantly, the FOH subscales of generalized anxiety disorder (0.739) and separation anxiety disorder (0.694) were highly correlated with the SCARED subscale of panic disorder (table 4). Multivariate linear analysis showed that higher age, female gender, MDI treatment type, longer duration of T1DM, higher frequency of hypoglycemia, passing out due to hypoglycemia, hypoglycemia while asleep and while awake, and hypoglycemia in front of friends and at school were the independent risk factors for the majority of the FOH and SCARED subscales (table 5). The total $\mathrm{R}^{2}$ statistics for these predictor variables for each dependent variable were as follows: behavior (0.398), worry (0.482), panic disorder (0.638), generalized anxiety disorder (0.471), separation anxiety disorder (0.521), social anxiety disorder (0.577), and significant school avoidance (0.652). 
Table 3. Mean comparisons of FOH, anxiety-related disorders and hypoglycemia history

\begin{tabular}{|c|c|c|c|c|c|c|c|}
\hline \multirow{2}{*}{$\begin{array}{l}\text { Hypoglycemia } \\
\text { history }\end{array}$} & \multicolumn{3}{|l|}{$\mathrm{FOH}$} & \multicolumn{4}{|l|}{ SCARED } \\
\hline & behavior & worry & $\begin{array}{l}\text { panic } \\
\text { disorder }\end{array}$ & $\begin{array}{l}\text { generalized } \\
\text { anxiety } \\
\text { disorder }\end{array}$ & $\begin{array}{l}\text { separation } \\
\text { anxiety } \\
\text { disorder }\end{array}$ & $\begin{array}{l}\text { social } \\
\text { anxiety } \\
\text { disorder }\end{array}$ & $\begin{array}{l}\text { significant } \\
\text { school } \\
\text { avoidance }\end{array}$ \\
\hline \multicolumn{8}{|c|}{ Frequency of hypoglycemic episodes } \\
\hline $1-2$ & $1.9 \pm 0.73$ & $2.35 \pm 0.77$ & $1 \pm 0.16$ & $0.82 \pm 0.24$ & $0.77 \pm 0.24$ & $1.21 \pm 0.24$ & $0.25 \pm 0.31$ \\
\hline$>11$ & $2 \pm 0.57^{c, e, f}$ & $1.83 \pm 1.19^{\mathrm{e}, \mathrm{f}}$ & $0.66 \pm 0.52^{c, d}$ & $0.63 \pm 0.46$ & $0.71 \pm 0.58^{\mathrm{e}, \mathrm{f}}$ & $0.71 \pm 0.62^{c, e, f}$ & $0.64 \pm 0.53^{c}$ \\
\hline \multicolumn{8}{|c|}{ Hypoglycemia as a big problem } \\
\hline Yes & $2.3 \pm 0.69$ & $2.41 \pm 0.89$ & $0.88 \pm 0.47$ & $0.69 \pm 0.41$ & $0.90 \pm 0.49$ & $0.95 \pm 0.53$ & $0.66 \pm 0.5$ \\
\hline No & $2.12 \pm 0.81$ & $2.19 \pm 0.98$ & $0.86 \pm 0.49$ & $0.77 \pm 0.36$ & $0.82 \pm 0.43$ & $0.95 \pm 0.61$ & $0.57 \pm 0.48$ \\
\hline \multicolumn{8}{|c|}{ Passed out due to hypoglycemia } \\
\hline Yes & $1.99 \pm 0.65$ & $2.44 \pm 0.92$ & $0.92 \pm 0.51$ & $0.73 \pm 0.4$ & $0.84 \pm 0.63$ & $0.84 \pm 0.64$ & $0.94 \pm 0.53$ \\
\hline \multicolumn{8}{|c|}{ Hypoglycemic episode while awake } \\
\hline Yes & $2.42 \pm 0.73$ & $2.6 \pm 0.71$ & $1 \pm 0.4$ & $0.78 \pm 0.35$ & $0.92 \pm 0.43$ & $1.1 \pm 0.52$ & $0.73 \pm 0.47$ \\
\hline No & $1.91 \pm 0.65^{\mathrm{g}}$ & $1.74 \pm 1.05^{\mathrm{g}}$ & $0.61 \pm 0.51^{\mathrm{g}}$ & $0.58 \pm 0.44^{\mathrm{g}}$ & $0.78 \pm 0.53^{\mathrm{g}}$ & $0.64 \pm 0.51^{\mathrm{g}}$ & $0.41 \pm 0.48^{g}$ \\
\hline \multicolumn{8}{|c|}{ Hypoglycemia in front of friends or strangers } \\
\hline Yes & $2.32 \pm 0.72$ & $2.42 \pm 0.84$ & $0.86 \pm 0.48$ & $0.66 \pm 0.38$ & $0.86 \pm 0.46$ & $0.96 \pm 0.57$ & $0.64 \pm 0.5$ \\
\hline No & $1.9 \pm 0.8^{\mathrm{g}}$ & $1.8 \pm 1.16^{\mathrm{g}}$ & $0.95 \pm 0.47$ & $0.9 \pm 0.4^{\mathrm{g}}$ & $0.94 \pm 0.48$ & $0.90 \pm 0.48$ & $0.54 \pm 0.47$ \\
\hline \multicolumn{8}{|c|}{ Hypoglycemia at school } \\
\hline Yes & $2.36 \pm 0.76$ & $2.35 \pm 0.81$ & $0.93 \pm 0.46$ & $0.72 \pm 0.38$ & $0.90 \pm 0.48$ & $1 \pm 0.55$ & $0.69 \pm 0.50$ \\
\hline No & $1.81 \pm 0.49 \mathrm{~g}$ & $2.1 \pm 1.2$ & $0.64 \pm 0.47 \mathrm{~g}$ & $0.68 \pm 0.45$ & $0.74 \pm 0.41$ & $0.57 \pm 0.37 \mathrm{~g}$ & $0.37 \pm 0.38^{g}$ \\
\hline
\end{tabular}

Values are presented as means \pm SD ( $t$ test, one-way analysis of variance and Tukey post hoc test). Group comparisons: ${ }^{\text {a }} 1-2$ vs. $3-6,{ }^{b} 1-2$ vs. $7-11,{ }^{c} 1-2$ vs. $>11,{ }^{\text {d }} 3-6$ vs. $7-11,{ }^{\mathrm{e}} 3-6$ vs. $>11,{ }^{\text {f }} 7-11$ vs. $>11,{ }^{\mathrm{g}}$ yes vs. no.

Table 4. Correlations between FOH and anxiety-related disorders

\begin{tabular}{|c|c|c|c|c|c|c|c|}
\hline & \multicolumn{2}{|l|}{$\mathrm{FOH}$} & \multicolumn{5}{|l|}{ SCARED } \\
\hline \multicolumn{8}{|l|}{$\mathrm{FOH}$} \\
\hline Behavior & 1 & & & & & & \\
\hline Worry & $0.423^{*}$ & 1 & & & & & \\
\hline Generalized anxiety disorder & 0.124 & $0.426^{*}$ & $0.739^{*}$ & 1 & & & \\
\hline Separation anxiety disorder & $0.269^{*}$ & $0.414^{*}$ & $0.694^{*}$ & $0.696^{*}$ & 1 & & \\
\hline Social anxiety disorder & $0.297^{*}$ & $0.240^{*}$ & $0.602^{*}$ & $0.477^{*}$ & $0.640^{*}$ & 1 & \\
\hline Significant school avoidance & $0.219^{*}$ & $0.351^{*}$ & $0.683^{*}$ & $0.494^{*}$ & $0.488^{*}$ & $0.240^{*}$ & 1 \\
\hline
\end{tabular}


Table 5. Results of multiple linear regression analysis

a $\mathrm{FOH}$

\begin{tabular}{|c|c|c|c|c|}
\hline \multirow[t]{2}{*}{ Variable } & \multicolumn{2}{|l|}{ Behavior } & \multicolumn{2}{|l|}{ Worry } \\
\hline & $\beta(95 \% \mathrm{CI})$ & $\mathrm{p}$ & $\beta(95 \% \mathrm{CI})$ & $\mathrm{p}$ \\
\hline Age & $0.563(0.274,0.852)$ & 0.000 & $0.691(0.359,1.022)$ & 0.000 \\
\hline Gender & $0.304(0.069,0.539)$ & 0.012 & $0.444(0.175,0.714)$ & 0.001 \\
\hline Education & $-0.208(-0.503,0.087)$ & 0.166 & $-0.766(-1.10,-0.427)$ & 0.000 \\
\hline Exercise & $-0.119(-0.349,0.110)$ & 0.307 & $-0.214(-0.478,0.050)$ & 0.111 \\
\hline Treatment type & $-0.095(-0.326,0.137)$ & 0.420 & $0.248(0.017,0.513)$ & 0.067 \\
\hline Duration & $0.316(0.064,0.567)$ & 0.014 & $0.304(0.016,0.593)$ & 0.039 \\
\hline $\mathrm{HbA}_{1 \mathrm{c}}$ & $0.021(-0.257,0.298)$ & 0.884 & $-0.317(-0.636,0.002)$ & 0.051 \\
\hline Frequency of hypoglycemic episodes & $-0.49(-0.147,0.050)$ & 0.331 & $-0.355(-0.468,-0.242)$ & 0.000 \\
\hline Hypoglycemia as a big problem & $-0.262(-0.489,-0.035)$ & 0.024 & $-0.105(-0.365,0.155)$ & 0.428 \\
\hline Passed out due to hypoglycemia & $0.502(0.271,0.734)$ & 0.000 & $-0.080(-0.345,0.186)$ & 0.556 \\
\hline Hypoglycemic episode while asleep & $-0.157(-0.448,0.133)$ & 0.287 & $-0.508(-0.842,-0.175)$ & 0.003 \\
\hline Hypoglycemic episode while awake & $-0.300(-0.550,-0.049)$ & 0.019 & $-0.602(-0.889,-0.314)$ & 0.000 \\
\hline Hypoglycemia in front of friends or strangers & $0.286(-0.042,0.613)$ & 0.087 & $-0.024(-0.400,0.351)$ & 0.898 \\
\hline Hypoglycemia at school & $-0.312(-0.593,-0.031)$ & 0.030 & $0.185(-0.138,0.507)$ & 0.259 \\
\hline
\end{tabular}

\section{b SCARED}

\begin{tabular}{|c|c|c|c|c|c|c|c|c|c|c|}
\hline \multirow[t]{2}{*}{ Variable } & \multicolumn{2}{|l|}{ Panic disorder } & \multicolumn{2}{|c|}{$\begin{array}{l}\text { Generalized anxiety } \\
\text { disorder }\end{array}$} & \multicolumn{2}{|c|}{$\begin{array}{l}\text { Separation anxiety } \\
\text { disorder }\end{array}$} & \multicolumn{2}{|l|}{$\begin{array}{l}\text { Social anxiety } \\
\text { disorder }\end{array}$} & \multicolumn{2}{|c|}{$\begin{array}{l}\text { Significant school } \\
\text { avoidance }\end{array}$} \\
\hline & $\beta(95 \% \mathrm{CI})$ & $\mathrm{p}$ & $\beta(95 \% \mathrm{CI})$ & $\mathrm{p}$ & $\beta(95 \% \mathrm{CI})$ & $\mathrm{p}$ & $\beta(95 \% \mathrm{CI})$ & $\mathrm{p}$ & $\beta(95 \% \mathrm{CI})$ & $\mathrm{p}$ \\
\hline Age & $\begin{array}{l}-0.254 \\
(0.371,1.35)\end{array}$ & 0.001 & $\begin{array}{l}-0.095 \\
(-0.238,0.049)\end{array}$ & 0.195 & $\begin{array}{l}-0.307 \\
(-0.469,-0.144)\end{array}$ & 0.000 & $\begin{array}{l}-0.240 \\
(-0.420,-0.059)\end{array}$ & 0.010 & $\begin{array}{l}-0.148 \\
(-0.294,-0.002)\end{array}$ & 0.047 \\
\hline Gender & $\begin{array}{l}0.183 \\
(0.67,0.299)\end{array}$ & 0.002 & $\begin{array}{l}0.060 \\
(-0.057,0.176)\end{array}$ & 0.315 & $\begin{array}{l}0.158 \\
(0.026,0.290)\end{array}$ & 0.019 & $\begin{array}{l}-0.014 \\
(-0.161,0.132)\end{array}$ & 0.848 & $\begin{array}{l}0.646 \\
(0.528,0.765) \\
\end{array}$ & 0.000 \\
\hline Education & $\begin{array}{l}0.249 \\
(0.103,0.395)\end{array}$ & 0.001 & $\begin{array}{l}0.027 \\
(-0.120,0.174) \\
\end{array}$ & 0.717 & $\begin{array}{l}0.307 \\
(0.141,0.472)\end{array}$ & 0.000 & $\begin{array}{l}0.470 \\
(0.285,0.654)\end{array}$ & 0.000 & $\begin{array}{l}-0.018 \\
(-0.167,0.131)\end{array}$ & 0.815 \\
\hline Exercise & $\begin{array}{l}-0.005 \\
(-0.118,0.109)\end{array}$ & 0.936 & $\begin{array}{l}0.29 \\
(-0.085,0.144) \\
\end{array}$ & 0.613 & $\begin{array}{l}-0.191 \\
(-0.320,-0.062)\end{array}$ & 0.004 & $\begin{array}{l}0.109 \\
(-0.035,0.252)\end{array}$ & 0.137 & $\begin{array}{l}-0.098 \\
(-0.214,0.018)\end{array}$ & 0.098 \\
\hline Treatment type & $\begin{array}{l}0.328 \\
(0.213,0.442)\end{array}$ & 0.000 & $\begin{array}{l}0.169 \\
(0.054,0.284)\end{array}$ & 0.004 & $\begin{array}{l}0.477 \\
(0.348,0.607)\end{array}$ & 0.000 & $\begin{array}{l}0.120 \\
(-0.025,0.264)\end{array}$ & 0.104 & $\begin{array}{l}0.050 \\
(-0.066,0.167)\end{array}$ & 0.395 \\
\hline Duration & $\begin{array}{l}0.288 \\
(0.164,0.413)\end{array}$ & 0.000 & $\begin{array}{l}0.417 \\
(0.292,0.542)\end{array}$ & 0.000 & $\begin{array}{l}0.475 \\
(0.334,0.616)\end{array}$ & 0.000 & $\begin{array}{l}0.394 \\
(0.237,0.551)\end{array}$ & 0.000 & $\begin{array}{l}0.222 \\
(0.095,0.349)\end{array}$ & 0.001 \\
\hline $\mathrm{HbA}_{1 \mathrm{c}}$ & $\begin{array}{l}-0.069 \\
(-0.207,0.068)\end{array}$ & 0.321 & $\begin{array}{l}0.030 \\
(-0.108,0.168)\end{array}$ & 0.671 & $\begin{array}{l}-0.094 \\
(-0.250,0.062)\end{array}$ & 0.234 & $\begin{array}{l}-0.527 \\
(-0.701,-0.354)\end{array}$ & 0.000 & $\begin{array}{l}0.142 \\
(0.002,0.283)\end{array}$ & 0.047 \\
\hline $\begin{array}{l}\text { Frequency of hypoglycemic } \\
\text { episodes }\end{array}$ & $\begin{array}{l}-0.143 \\
(-0.191,-0.94)\end{array}$ & 0.000 & $\begin{array}{l}-0.56 \\
(-0.105,-0.007)\end{array}$ & 0.026 & $\begin{array}{l}-0.55 \\
(-0.110,0.000)\end{array}$ & 0.052 & $\begin{array}{l}-0.021 \\
(-0.082,0.041)\end{array}$ & 0.505 & $\begin{array}{l}-0.005 \\
(-0.055,0.044)\end{array}$ & 0.831 \\
\hline $\begin{array}{l}\text { Hypoglycemia as a big } \\
\text { problem }\end{array}$ & $\begin{array}{l}-0.115 \\
(-0.227,-0.002)\end{array}$ & 0.045 & $\begin{array}{l}0.59 \\
(-0.054,0.172)\end{array}$ & 0.305 & $\begin{array}{l}-0.112 \\
(-0.239,0.15)\end{array}$ & 0.084 & $\begin{array}{l}0.013 \\
(-0.129,0.155) \\
\end{array}$ & 0.854 & $\begin{array}{l}-0.168 \\
(-0.282,-0.053)\end{array}$ & 0.004 \\
\hline $\begin{array}{l}\text { Passed out due } \\
\text { to hypoglycemia }\end{array}$ & $\begin{array}{l}-0.086 \\
(-0.200,-0.29)\end{array}$ & 0.142 & $\begin{array}{l}0.056 \\
(-0.059,0.171)\end{array}$ & 0.337 & $\begin{array}{l}0.189 \\
(0.059,0.319)\end{array}$ & 0.005 & $\begin{array}{l}0.108 \\
(-0.037,0.253)\end{array}$ & 0.144 & $\begin{array}{l}-0.271 \\
(-0.388,-0.154)\end{array}$ & 0.000 \\
\hline $\begin{array}{l}\text { Hypoglycemic episode } \\
\text { while asleep }\end{array}$ & $\begin{array}{l}-0.108 \\
(-0.252,0.036)\end{array}$ & 0.140 & $\begin{array}{l}-0.253 \\
(-0.397,-0.108)\end{array}$ & 0.001 & $\begin{array}{l}-0.274 \\
(-0.437,-0.111)\end{array}$ & 0.001 & $\begin{array}{l}-0.148 \\
(-0.330,0.33)\end{array}$ & 0.109 & $\begin{array}{l}-0.059 \\
(-0.206,0.088)\end{array}$ & 0.426 \\
\hline $\begin{array}{l}\text { Hypoglycemic episode } \\
\text { while awake }\end{array}$ & $\begin{array}{l}-0.318 \\
(-0.442,-0.194)\end{array}$ & 0.000 & $\begin{array}{l}-0.206 \\
(-0.331,-0.081)\end{array}$ & 0.001 & $\begin{array}{l}0.028 \\
(-0.113,0.168)\end{array}$ & 0.700 & $\begin{array}{l}-0.388 \\
(-0.545,-0.231)\end{array}$ & 0.000 & $\begin{array}{l}-0.015 \\
(-0.142,0.111)\end{array}$ & 0.810 \\
\hline $\begin{array}{l}\text { Hypoglycemia in front } \\
\text { of friends or strangers }\end{array}$ & $\begin{array}{l}0.595 \\
(0.433,0.754)\end{array}$ & 0.000 & $\begin{array}{l}0.537 \\
(0.374,0.669)\end{array}$ & 0.000 & $\begin{array}{l}0.321 \\
(0.138,0.505)\end{array}$ & 0.001 & $\begin{array}{l}0.362 \\
(0.157,0.566)\end{array}$ & 0.001 & $\begin{array}{l}0.303 \\
(0.138,0.469)\end{array}$ & 0.000 \\
\hline Hypoglycemia at school & $\begin{array}{l}-0.284 \\
(-0.423,-0.145)\end{array}$ & 0.000 & $\begin{array}{l}-0.177 \\
(-0.317,-0.037)\end{array}$ & 0.013 & $\begin{array}{l}-0.232 \\
(-0.389,-0.074)\end{array}$ & 0.004 & $\begin{array}{l}-0.367 \\
(-0.543,-0.192)\end{array}$ & 0.000 & $\begin{array}{l}-0.139 \\
(-0.281,0.003)\end{array}$ & 0.055 \\
\hline
\end{tabular}

$* p<0.05$ considered significant.

Fear of Hypoglycemia and Anxiety-Related Disorders 


\section{Discussion}

The findings of this study confirmed that hypoglycemia is a risk factor for developing fear and psychological problems in children and adolescence. The FOH involves both the negative acute health consequences of an episode and fear of social reprisal for the behavioral, motor and cognitive changes that occur during an episode $[16,17]$. This had also been reported in patients with frequent hypoglycemia, with linkages to anxiety and phobia about future hypoglycemic events $[17,18]$. Further studies have shown that as children with T1DM grew into young adulthood $42 \%$ developed at least one episode of psychiatric disorder, the most common being depressive disorders $(26 \%)$ followed by anxiety disorders $(20 \%)$ and behavior disorders $(16 \%)[19,20]$. Therefore, adolescents with diabetes are at particular risk for developing psychological disorders. However, while attention to psychological disorders in adolescents with T1DM is important, limited research has focused on psychological functioning and $\mathrm{FOH}$ in the Arab population, particularly in Saudi Arabia.

Numerous risk factors have been reported for psychological disorders in adolescents with T1DM that include a higher prevalence in females than males and an expression of greater concern for hypoglycemia in the female gender [21,22], which is confirmed by the present study findings. Other studies have also reported that social anxiety involves a fear and avoidance of social situations as well as self-critical evaluations while in the presence of others. This is not unexpected since social anxiety is prevalent during adolescence, a period marked by social pressures and the desire to belong to a social group [22]. In addition, studies have consistently found that females endorse more diabetes-related worries, less satisfaction and poorer perceptions of their own health than males [5, 23, 24]. These results are important from a clinical viewpoint but are not surprising in that they are consistent with data on anxiety disorders in general. We can only speculate on why females show higher FOH than men. Possible hypotheses include biological differences, with females in general having a genetic predisposition towards being more fearful, as well as cultural aspects, that is, it might be more acceptable for female to express SCARED and $\mathrm{FOH}$. In multivariate analysis we also found that female gender was an independent risk factor for higher SCARED and $\mathrm{FOH}$ subscales.

In the present study, we found that the mean scores for the majority of FOH and SCARED subscales were higher in the older age group (16-18 years) compared with the younger age group (13-15 years). The age differences of FOH in youths with T1DM have not yet been well studied. For example, Gonder-Frederick et al. [5] stated that no studies had examined $\mathrm{FOH}$ across a broad range of age groups in youths, often because of the relatively small numbers of participants and the restricted age ranges of the youths. In the present study we found higher levels of FOH and SCARED in those under MDI treatment (compared with the insulin pump) and in those with longer duration of T1DM ( $>7$ years). Similarly, using multivariate linear analysis we also found that treatment type and longer duration were independent risk factors for the majority of the FOH and SCARED subscales. Previous research has reported that, with FOH increasing with the duration of diabetes, hypoglycemia becomes a significant problem as many patients experience weakening of hypoglycemic warning symptoms [25].

Many studies have indicated that adolescent diabetes management is associated with better metabolic control, and diabetes management tasks are related to better psychological health and self-care behavior [26, 27]. However, maintaining $\mathrm{HbA}_{1 \mathrm{c}}$ as close to normal as possible increases the risk of hypoglycemia, and the $\mathrm{FOH}$ is likely to affect diabetes management [28]. Indeed, hypoglycemia is the most likely adverse event associated with insulin treatment in T1DM [17]. The FOH is likely to result from the fear of physical consequences and the fear of social embarrassment related to the behavioral, motor and emotional changes that may occur during an episode of hypoglycemia [17]. It is notable that there were no significant differences observed between the $\mathrm{HbA}_{1 \mathrm{c}}$ control and FOH and SCARED in the present study except the school avoidance and social anxiety subscale scores. Previous research has also demonstrated inconsistency related to the association between $\mathrm{FOH}$ and glycemic control [17]. The mental mechanisms associated with barriers to diabetes management and the role of FOH need to be better understood. The behavior contributing to poor glycemic control needs to be recognized to develop adequate strategies and support programs for adolescents with T1DM [17]. It should be noted here that it is possible to minimize hypoglycemic risks by understanding the physiological counter regulatory responses and aggressively monitoring glycemic therapy. Education concerning the self-monitoring of blood glucose, diet, physiological insulin replacement, medication, and lifestyle are important to maintain good glycemic control, avoid hypoglycemia and prevent long-term complications.

Increasingly, evidence is emerging that a higher frequency of hypoglycemia was the most important factor 
associated with FOH $[13,29]$. In our study we also found that adolescents with higher frequencies of hypoglycemia showed significantly higher scores of FOH and SCARED subscales except for the separation anxiety disorder. Similarly, we found that adolescents with hypoglycemia showed higher school avoidance, $\mathrm{FOH}$ while asleep and awake, and $\mathrm{FOH}$ in front of friends or strangers and during school time; these findings are consistent with several previous studies $[13,30]$.

The construct of FOH in adolescents and SCARED has received relatively limited scientific attention. In our study we found that the FOH subscale of behavior correlated with all the subscales of SCARED except that of generalized anxiety disorder. Similarly, the FOH subscale of worry significantly correlated with all the subscales of SCARED. This finding supports the hypothesis that developing $\mathrm{FOH}$ is a risk factor for other types of anxiety $[17,18]$. Also, numerous studies have reported that there are a number of factors that relate to whether an individual is likely to develop FOH, including history of hypoglycemia, frequency of hypoglycemia, impaired awareness of hypoglycemia, age, gender, duration of diabetes, and length of time since first insulin treatment $[13,17]$. The multivariate linear analysis of our study also revealed that higher age, female gender, MDI treatment type, longer duration of T1DM, higher frequency of hypoglycemia, passing out due to hypoglycemia, hypoglycemia while asleep and awake, and hypoglycemia in front of friends and at school were independent risk factors for the majority of the FOH and SCARED subscales.

The major limitations of this study were the following: (1) there were a limited number of risk factors and sociodemographic factors examined, (2) the study was performed at a single center and (3) there was no control group with which to compare the study group. Further research is needed to address the limitations indicated in the study as well as how $\mathrm{FOH}$ arises and the individual variables which predict its development. Well-designed research is required to better understand the behavioral and medical impact of FOH and interventions to reduce it.

In conclusion, the study indicated that the strongest determinants of higher risk for the majority of the $\mathrm{FOH}$ and SCARED subscales were higher age, female gender, MDI treatment, longer duration of T1DM, higher frequency of hypoglycemia, passing out due to hypoglycemia, hypoglycemia while asleep and awake, and hypoglycemia in front of friends and at school. Further, the study showed that the FOH subscale of behavior correlated with all the subscales of SCARED except that of generalized anxiety disorder. Similarly, the FOH subscale of worry significantly correlated with all the subscales of SCARED. Overall, the study results revealed that FOH and SCARED scores were higher among Saudi adolescents with T1DM. It is important that adolescents with a higher risk of hypoglycemia and their parents/caregiver are educated about the prevention and treatment of hypoglycemia in order to alleviate FOH and SCARED during activities of daily living.

\section{Acknowledgment}

We thank Dr. L.A. Gonder-Frederick, University of Virginia, for her permission to use the questionnaire $(\mathrm{FOH})$ in our study.

\section{Disclosure Statement}

The authors have no conflicts of interest to disclose.

\section{References}

Fear of Hypoglycemia and Anxiety-Related Disorders 
9 Cryer PE, Davis SN, Shamoon H: Hypoglycemia in diabetes. Diabetes Care 2003;26:19021912.

-10 Al-Herbish AS, El-Mouzan MI, Al-Salloum AA, et al: Prevalence of type 1 diabetes mellitus in Saudi Arabian children and adolescents. Saudi Med J 2008;29:1285-1288.

-11 Silverstein J1, Klingensmith G, Copeland K, et al: Care of children and adolescents with type 1 diabetes: a statement of the American Diabetes Association. Diabetes Care 2005;28: 186-212.

12 Lukacs A, Varga B, Kiss-Toth E, et al: Factors influencing the diabetes-specific health-related quality of life in children and adolescents with type 1 diabetes mellitus. J Child Health Care 2014;18:253-260.

13 Gonder-Frederick LA, Fisher CD, Ritterband LM, et al: Predictors of fear of hypoglycemia in adolescents with type 1 diabetes and their parents. Pediatr Diabetes 2006; 7:215-222.

14 Birmaher B, Khetarpal S, Brent D, et al: The Screen for Child Anxiety-Related Emotional Disorders (SCARED): scale construction and psychometric characteristics. J Am Acad Child Adolesc Psychiatry 1997;36:545-553.

15 Hale WW, 3rd, Crocetti E, Raaijmakers QA, et al: A meta-analysis of the cross-cultural psychometric properties of the Screen for Child Anxiety-Related Emotional Disorders (SCARED). J Child Psychol Psychiatry 2011; 52:80-90.
16 Hislop AL, Fegan PG, Schlaeppi MJ, et al: Prevalence and associations of psychological distress in young adults with type 1 diabetes. Diabet Med 2008;25:91-96.

17 Wild D, von Maltzahn R, Brohan E, et al: A critical review of the literature on fear of hypoglycemia in diabetes: implications for diabetes management and patient education. $\mathrm{Pa}$ tient Educ Couns 2007;68:10-15.

18 Costea M, Ionescu-Tirgoviste C, Cheta D, et al: Fear of hypoglycemia in type 1 (insulindependent) diabetic patients. Rom J Intern Med 1993;31:291-295.

19 Kovacs M, Goldston D, Obrosky DS, et al: Psychiatric disorders in youths with IDDM: rates and risk factors. Diabetes Care 1997;20: 36-44.

20 Whittemore R, Jaser S, Guo J, et al: A conceptual model of childhood adaptation to type 1 diabetes. Nurs Outlook 2010;58:242-251.

21 Gjerlow E, Bjorgaas MR, Nielsen EW, et al: Fear of hypoglycemia in women and men with type 1 diabetes. Nurs Res 2014;63:143-149.

22 Erwin BA, Turk CL, Heimberg RG, et al: The Internet: home to a severe population of individuals with social anxiety disorder? J Anxiety Disord 2004;18:629-646.

23 Faulkner MS: Quality of life for adolescents with type 1 diabetes: parental and youth perspectives. Pediatr Nurs 2003;29:362-368.
24 Valenzuela JM1, Patino AM, McCullough J, et al: Insulin pump therapy and health-related quality of life in children and adolescents with type 1 diabetes. J Pediatr Psychol 2006;31: 650-660.

25 Pramming S, Thorsteinsson B, Bendtson I, et al: Symptomatic hypoglycaemia in 411 type 1 diabetic patients. Diabet Med 1991;8:217222

26 Anderson BJ, Vangsness L, Connell A, et al: Family conflict, adherence, and glycaemic control in youth with short duration Type 1 diabetes. Diabet Med 2002;19:635-642.

27 Palmer DL, Berg CA, Wiebe DJ, et al: The role of autonomy and pubertal status in understanding age differences in maternal involvement in diabetes responsibility across adolescence. J Pediatr Psychol 2004;29:35-46.

28 Hypoglycemia in the Diabetes Control and Complications Trial. The Diabetes Control and Complications Trial Research Group. Diabetes 1997;46:271-286.

- 29 Anderbro T1, Amsberg S, Adamson U, et al: Fear of hypoglycaemia in adults with type 1 diabetes. Diabet Med 2010;27:1151-115-8.

30 Di Battista AM, Hart TA, Greco L, et al: Type 1 diabetes among adolescents: reduced diabetes self-care caused by social fear and fear of hypoglycemia. Diabetes Educ 2009;35:465475 . 